\section{P11.09 STI PREVENTION IN PUBLIC HEALTH SERVICES FOR TRANSGENDER WOMEN IN GUATEMALA}

${ }^{1} \mathrm{R}$ Mendizabal-Burastero*, ' C Galindo-Arandi, 'I Loya-Montiel, ${ }^{2} \mathrm{~J}$ Garcia, ${ }^{1} \mathrm{~S}$ MoralesMiranda. 'HIV Unit, Center for Health Studies, Universidad Del Valle de Guatemala; ${ }^{2}$ National Reference Laboratory, Ministry of Health, Guatemala

\subsection{6/sextrans-2015-052270.457}

Introduction Transgender women (TG) have the highest HIV prevalence in Guatemala (23.9\%). Since 2007, the STI Sentinel Surveillance and Control (VICITS) strategy has provided STI diagnosis and treatment, behavioural change interventions and condoms to key populations in coordination with the Ministry of Health. We present key biological and behavioural characteristics of TG who attended VICITS clinics in Guatemala between 2007 and 2014

Methods TG $\geq 18$ years old (yo), who had sex with a man in the last year and attended one of four VICITS clinics in Guatemala were included in the analysis. HIV and syphilis infection were diagnosed according to national guidelines. Sociodemographic data, risk behaviour, and biological data were collected. Data analysis was conducted in Stata 11.0.

Results A total of 151 TG sought care at least once during 2007-2014 and 366 consultations were provided. Median age was 27 yo (IQR 23-34), 76.3\% had secondary education or more, $77.7 \%$ reported sex work in the last year $(75 \%$ for more than one year), $40.4 \%$ reported drug use at least once in lifetime with half of them reporting active use in the last 30 days cocaine was the most common drug. Condom use in the last sexual relationship was $85.4 \%, 89 \%$ of the TG reported receptive anal sex and $88.8 \%$ oral sex in the last 30 days. Approximately $7.2 \%$ had a previous HIV diagnosis and $4.2 \%$ were newly diagnosed for a total HIV prevalence of $11 \%$. Of those tested for syphilis, 3.9\% had active syphilis infection.

Conclusion We found high HIV and active syphilis prevalence among TG, suggesting that additional efforts and strategies for prevention among this group are needed. Due to the high proportion of receptive anal sex reported, surveillance of anal STI should be prioritised and conducted to provide appropriate and timely data to control the epidemic among key populations.

Disclosure of interest statement We declare that we have no conflicts of interest.

\section{P11.10 ANAL AND OROPHARYNGEAL STI SURVEILLANCE AMONG MEN WHO HAVE SEX WITH MEN IN GUATEMALA}

${ }^{1} \mathrm{R}$ Mendizabal-Burastero, ${ }^{1} \mathrm{C}$ Vargas, ${ }^{1} \mathrm{C}$ Galindo-Arandi, ${ }^{1} \mathrm{I}$ Loya-Montiel, ${ }^{2} \mathrm{C}$ Valenzuela, ${ }^{1} S$ Morales-Miranda. 'HIV Unit, Center for Health Studies, Universidad Del Valle de Guatemala; ${ }^{2}$ National Reference Laboratory, Ministry of Health, Guatemala

\subsection{6/sextrans-2015-052270.458}

Introduction Sentinel Surveillance and STI Control (VICITS) strategy is implemented in public health clinics for key populations, including men who have sex with men (MSM) in Guatemala. Although oral and anal sexually transmitted infections (STI) are important public health issues, they are not included in national treatment guidelines. Evidence of disease burden is needed for decision making. We analysed STI prevalence in oral, anal and urethral samples among MSM who attended VICITS clinics in Guatemala in 2014.

Methods Clients were male, $\geq 18$ years old, who reported sexual relations with another man in the last 12 months, and who was seen at one of four VICITS clinics between June-December 2014. Sociodemographic data, risk behaviour, and biological data were abstracted from routine clinical intake forms stored in the in VICITS information system. Laboratory results from urethral, anal and oropharyngeal samples were collected. Digene Hybrid Capture II (Qiagen) was used for detection of C. trachomatis (CT) and N. gonorrhoeae (NG). Data analysis was performed using Stata 11.0.

Results A total of 524 MSM were included in the analysis. The median age was 25 years old (IQR 21-30), 67.4\% reported receptive anal sex in the last 30 days, and $56.6 \%$ reported condom use at last receptive anal sex. About 30\% reported at least one STI and 4.6\% reported at least 2 STIs in the last 3 months. The prevalence of anal CT was $4.7 \%$, anal NG was $4.4 \%$, oral CT was $2.2 \%$, oral NG was $25.4 \%$, urethral CT was $1.9 \%$, and urethral NG was $4.1 \%$. Overall, $77.7 \%$ of infections were caused by NG.

Conclusion We found a high prevalence of NG, especially in oral and anal samples among MSM attending VICITIS clinics. Our findings underscore the need to include anal and oral STIs in national treatment guidelines. Innovative strategies to increase adoption of condom use in anal and oral sex among MSM and the evaluation of resistance profile for NG are urgently needed in Guatemala.

Disclosure of interest statement We declare that we have no conflicts of interest.

\section{P11.11 HIGH RATES OF CO-OCCURRING SEXUALLY TRANSMISSIBLE INFECTIONS AMONG MEN WHO HAVE SEX WITH MEN ATTENDING HIGH-CASELOAD GENERAL PRACTICE AND SEXUAL HEALTH SERVICES IN VICTORIA}

${ }^{1} \mathrm{D}$ Leitinger*, ${ }^{1,2} \mathrm{~A}$ Wilkinson, ${ }^{1} \mathrm{~N}$ Bartnik, ${ }^{2} \mathrm{C}$ Fairley, ${ }^{3} \mathrm{BK}$ Tee, ${ }^{4} \mathrm{~N}$ Roth, ${ }^{5} \mathrm{D}$ Leslie, ${ }^{1} \mathrm{C}$ El-Hayek, ${ }^{1} \mathrm{M}$ Hellard, ${ }^{1} \mathrm{M}$ Stoové. ${ }^{1}$ Burnet Institute; ${ }^{2}$ School of Public Health and Preventive Medicine, Monash University; ${ }^{3}$ Melbourne Sexual Health Centre; ${ }^{4}$ Centre Clinic; ${ }^{5}$ Prahran Market Clinic; ${ }^{6}$ Victorian Infectious Diseases Reference Laboratory (VIDRL)

\subsection{6/sextrans-2015-052270.459}

Introduction Sexually transmissible infection (STI) screening and treatment is a health priority for gay, bisexual and other men who have sex with men (GBM). Current Australian guidelines recommend frequent syphilis serology and screening for pharyngeal, anorectal and urogenital chlamydia and gonorrhoea infection, in addition to routine HIV testing. However, evidence from recent community surveys that many Australian GBM access only some of these tests suggests concurrent infections may remain undetected.

Methods To better understand the epidemiology of co-occurring curative STIs, we calculated the proportion of positive tests for one or more STI among GBM attending two sexual health services and two gay-friendly high-caseload general practices in Victoria between 2007 and 2013. Among positive tests for chlamydia or gonorrhoea, we calculated the proportion positive at multiple anatomical sites. We examined demographic and risk behaviour associations with co-occurring STIs and positivity at multiple anatomical sites using adjusted logistic regression.

Results There were 70977 test events between 2007 and 2013; $8316(11.7 \%)$ were positive for at least one STI and of these, $792(9.5 \%)$ were positive for more than one STI. Younger age (16-29 years) $(\mathrm{aOR}=1.7,95 \% \mathrm{CI}=1.1-2.7)$, being HIV-positive $(\mathrm{aOR}=2.2,95 \% \mathrm{CI}=1.5-3.1)$ and reporting inconsistent condom use $(\mathrm{aOR}=1.3,95 \% \mathrm{CI}=1.0-1.6)$ were associated with testing positive for co-occurring infections. 
Of 7584 test events positive for chlamydia or gonorrhoea, 1028 (13.6\%) were positive for either infection at multiple sites. Being aged $16-29(\mathrm{aOR}=2.9,95 \% \mathrm{CI}=1.8-4.6)$ or $30-39$ $(\mathrm{aOR}=2.4,95 \% \mathrm{CI}=1.5-3.9)$ was associated with testing positive for either infection at multiple sites.

Conclusion The high prevalence of co-occurring STIs and infection at multiple anatomical sites supports national guidelines for frequent comprehensive testing for GBM. These results support the promotion of comprehensive STI testing for GBM, including in lower-caseload settings alongside taking appropriate sexual histories, and underline the importance of structural efforts to maximise access to comprehensive testing for GBM.

Disclosure of interest statement All authors have no conflicts to declare. The authors gratefully acknowledge patients and participating clinics and laboratories for the ongoing data contribution. The authors acknowledge Drs Norm Roth and BK Tee for their ongoing support of surveillance work at the Burnet Institute. The Victorian Department of Health funds ongoing surveillance projects within the Burnet Institute. The authors would like to acknowledge the NHMRC who provide funding to Anna Wilkinson as a public health scholarship recipient. Mark Stoové is supported by an NHMRC Career Development Fellowship. The authors gratefully acknowledge the contribution to this work of Victorian Operational Infrastructure Support Program received by the Burnet Institute.

\section{P11.12 PHARYNGEAL NEISSERIA GONORRHOEAE AND CHLAMYDIA TRACHOMATIS IN MEN AND WOMEN WITH A HISTORY OF RECEPTIVE ORAL AND ANAL INTERCOURSE}

${ }^{1} \mathrm{CS}$ Danby*, ${ }^{2} \mathrm{LA}$ Cosentino, ${ }^{2} \mathrm{LK}$ Rabe, ${ }^{2} \mathrm{CL}$ Priest, ${ }^{2} \mathrm{KC}$ Damare, ${ }^{2} \mathrm{IS}$ Macio, ${ }^{2} \mathrm{LA}$ Meyn, ${ }^{1,2} \mathrm{HC}$ Wiesenfeld, 1,2 $\mathrm{SL}$ Hillier. 'University of Pittsburgh, Department of Obstetrics, Gynecology and Reproductive Sciences; ${ }^{2}$ Magee-Womens Research Institute (2), Pittsburgh, $P A$, USA

\subsection{6/sextrans-2015-052270.460}

Introduction Both women and men who have sex with men (MSM) report frequent receptive oral sex, but there are no FDA approved tests for detection of Neisseria gonorrhoeae (GC) and Chlamydia trachomatis (CT) from pharyngeal swabs. The objective of this study was to evaluate the number of pharyngeal GC and CT infections, and to assess the sensitivity of GC culture.

Methods Women $(\mathrm{n}=172)$ and MSM $(\mathrm{n}=222)$ reporting a lifetime history of both receptive oral and anal sex completed a structured questionnaire and clinicians collected swab samples from the pharynx and anorectum. Vaginal swabs were obtained from women and urine samples from men. Testing for CT and GC was performed using Cepheid Xpert CT/NG (Xpert) and Gen-Probe Aptima (AC2). True positives were defined if both AC2 and Xpert were positive, if GC culture was positive, or if either AC2 or Xpert were positive and confirmatory tests APTIMA CT or APTIMA GC were positive.

Results Identification of GC and/or CT at any site (pharyngeal, rectal or genitourinary) occurred in 78/222 (35\%) males and 25/ $172(14.5 \%)$ of females. Only $8(2 \%)$ of pharyngeal swab samples were positive for CT, while 4 women $(2.3 \%)$ and 37 (16.7\%) men tested positive for pharyngeal GC based on NAAT. GC culture of the pharynx was performed for 373 of participants in the study, and was positive in 13 (3.5\%), for a sensitivity of $32 \%$ for culture vs NAAT. Overall, 17/55 (31\%) of the total GC infections in men would have been missed without NAAT pharyngeal testing.

Conclusion Men who report a lifetime history of receptive oral intercourse have high rates of pharyngeal GC, most of which will remain undetected unless NAAT is used. The frequency of pharyngeal infection due to CT is low even among people having high overall rates of infection.

Disclosure of interest Reagents for CT/GC testing were provided for by Cepheid and Hologic.

\section{P11.13 EXTRA-GENITAL AND URETHRAL CHLAMYDIA TRACHOMATIS AND NEISSERIA GONORRHEAE PREVALENCE AND ASSOCIATED RISK FACTORS IN MEN WHO HAVE SEX WITH MEN AND TRANSGENDER WOMEN IN LIMA, PERU}

1J Cabeza*, 'ER Segura, ${ }^{2} S M$ Montano, ${ }^{3} J R$ Lama, ${ }^{3} J$ Sanchez, ${ }^{1} J D$ Klausner, ${ }^{1} J L$ Clark. ${ }^{1}$ Department of Medicine, Division of Infectious Diseases and Center for World Health, David Geffen School of Medicine at University of California at Los Angeles, Los Angeles, California, USA; ${ }^{2}$ US Naval Medical Research Unit 6, Lima, Peru; ${ }^{3}$ Asociación Civil Impacta Salud Y Educación, Lima, Peru

\subsection{6/sextrans-2015-052270.461}

Introduction Data regarding extra-genital Neisseria gonorrheae (NG) and Chlamydia trachomatis (CT) in men who have sex with men (MSM), and transwomen (TW) in Latin America is limited. We determined the prevalence of pharyngeal, rectal, and urethral NG and CT among MSM, and TW and associated risk factors and behaviours.

Methods We used data from the Expedited Partner Therapy Screening Study of men who have sex with men (MSM) and transgender women (TW) in Lima, Peru. Participants completed a CASI survey regarding socio-demographic data, sexual risk behaviours, and STD symptoms. Anal and pharyngeal swabs and urine specimens collected by the study physician were tested for CT and NG using NAAT (Aptima GenProbe).

Results We screened 834 participants (551-gay identified men, 264 heterosexual/bisexual men and 19 TW) for CT and NG between October 2012 and July 2014. The median age of participants was 27 years (range 18-70). CT prevalence was 3.5\% in urine, $4.5 \%$ in pharyngeal and $15.6 \%$ in rectal specimens. NG prevalence was $2.7 \%$ in urine, $9.4 \%$ in pharyngeal and $8.3 \%$ in rectal specimens. Approximately $75 \%$ of participants with rectal infections reported no symptoms in the previous 30 days. More than $92 \%$ of extra-genital CT infections and $83 \%$ of extra-genital NG infections were identified in participants with negative urine test results. Younger age was strongly associated with increased prevalence of both pharyngeal NG $(\mathrm{p}=0.054)$ and rectal CT $(p=0.008)$ and NG infections $(p=0.002)$. Receptive anal intercourse was associated with higher prevalence of rectal CT infection $(p=0.012)$. Pharyngeal NG $(p=0.005)$ was more frequent in participants reporting multiple partners.

Conclusion Extragenital CT and NG infection is highly prevalent, usually asymptomatic and in a majority of cases, associated with negative urine tests among MSM and TW in Peru. Routine screening at multiple anatomic sites using NAAT should be performed. 